1 MaCaron MM, Wood JD. The cocaine "body packer" syndrome - diagnosis and treatment. $\mathcal{F A M A} 1983 ; 250$ : $1417-20$.

2 Caruana DS, Weinbach B, George D, Gardner LB. Cocainepacket ingestion. Ann Intern Med 1984; 100: 73-4.

3 Lancshire MJR, Legg PK, Lowe M, Davidson SA, Ellis BW.

Surgical aspects of international drug smuggling. $B M \mathcal{F}$ Surgical aspects of
$1988 ; 296,1035-7$.
4 Dassel PM, Punjabi E. Ingested marihuana filled balloons. Gasteroenterology 1979; 76, 166-9.

5 Horrocks AW. Abdominal radiography in suspected 'body packers'. Clin Radiol 1992; 45: 322-5.

\title{
An unusual neurological problem in a patient admitted for acute myocardial infarction
}

\author{
AD Kelion, M Shahi, JA Bell
}

Department of Cardiology, Battle Hospital, Oxford Road, Reading, Berks RG3 1AG, UK AD Kelion

M Shahi

JA Bell

Correspondence to $\mathrm{Dr}$ AD Kelion, Department of Cardiology, John Radcliffe Hospital, Oxford OX3 9DU, UK

Accepted 12 December 1996
A 70-year old man was admitted with unstable angina, and five days later was thrombolysed with intravenous streptokinase for an acute anterolateral myocardial infarction. After another four days he developed a frontal headache and photophobia, followed a few hours later by a sudden onset of complete ophthalmoplegia of the left eye, a complete left ptosis, and a fixed and dilated left pupil. No visual field defect or loss of visual acuity was evident. Computed tomography (CT) of the brain was reported as being unremarkable, although the cavernous sinuses were not properly visualised. The cerebrospinal fluid was clear with a protein level of $0.73 \mathrm{~g} / \mathrm{l}$.

On day 12 of the admission he became drowsy, pyrexial, and mildly hypotensive. Blood tests, electrocardiogram, chest X-ray, and echocardiogram remained unchanged, and an abdominal ultrasound scan was unremarkable. Urine and several sets of blood cultures were sterile. Following one unit of colloid, he was kept gently hydrated with intravenous crystalloid. He was treated for presumed sepsis with broad-spectrum antibiotics, and acyclovir was administered to cover the possibility of a Herpes simplex encephalitis. Unfortunately his condition remained unchanged, and on day 17 of the admission he developed acute pulmonary oedema and died a few hours later on the intensive care unit.

\section{Questions}

1 What was the diagnosis?

2 How might the diagnosis have been confirmed?

3 What was the appropriate management? 
Answers

QUESTION 1

Pituitary apoplexy caused by haemorrhage into a previously asymptomatic adenoma.

An autopsy confirmed the presence of an anterolateral myocardial infarction, severe twovessel coronary disease, and pulmonary oedema. The pituitary gland was greatly enlarged at $35 \times 25 \times 20 \mathrm{~mm}$, and comprised soft pink tissue with an $8 \mathrm{~mm}$ area of recent haemorrhage. The appearance was that of a tumour that had breached the hypophyseal fossa and extended into the left cavernous sinus to surround the left internal carotid artery. Histological examination identified the tumour as an acidophil (eosinophil) adenoma.

Pituitary apoplexy is a rare condition which usually results from haemorrhage into a macroadenoma. Of pituitary tumours coming to surgery, only about $5 \%$ have caused clinical apoplexy. ${ }^{1}$ The tumour is usually an unsuspected nonsecretory macroadenoma. Several iatrogenic precipitants of haemorrhage have been described, including long- and short-term anticoagulation. To our knowledge, however, it has not previously been described as a complication of thrombolysis for myocardial infarction. The causes of pituitary apoplexy are summarised in box 1 .

Pituitary apoplexy may present with a variety of neurological symptoms and signs (box 2). ${ }^{2}$ Loss of hormonal secretion, particularly adrenocorticotropin, can rapidly become life-threatening.

QUESTION 2

High-resolution unenhanced coronal CT scanning of the pituitary fossa, or $T_{1}$-weighted magnetic resonance imaging would have confirmed the diagnosis. The former may be better within the first few days, but the latter is more sensitive for detecting or following the haemorrhage subacutely. ${ }^{3,4}$ A standard CT brain scan is poor at imaging the cavernous sinuses. Appropriate imaging was not undertaken in the above case because it was not envisaged that a remediable lesion would be found.

QUESTION 3

High-dose corticosteroid (eg, dexamethasone $2 \mathrm{mg}$ qid) may be required acutely to reduce cerebral oedema, and conventional steroid replacement therapy should be continued until the clinical situation is stable. Early surgical decompression is indicated for persistent

1 Bonicki W, Kasperlik-Zaluska A, Koszewski W, Zgliczynski W, Wislawski J. Pituitary apoplexy: endocrine, surgical, and oncological emergency. Incidence, clinical course and treatment with reference to 799 cases of pituitary adenomas. Acta Neurochir Wien 1993; 120: 118-22.

2 Bills DC, Meyer FB, Laws ER Jr, et al. A retrospective analysis of pituitary apoplexy. Neurosurgery 1993; 33: 602-8.

3 L'Huillier F, Combes C, Martin N, Leclerc X, Pruvo JP, Gaston A. MRI in the diagnosis of so-called pituitary apoplexy: seven cases. F Neuroradiol 1989; 16: 221 - 37.

\section{Causes of pituitary apoplexy}

- haemorrhage into an adenoma: precipitants include trauma, pituitary irradiation, pneumoencephalography, carotid angiography, pituitary stimulation tests, cardiopulmonary bypass, and anticoagulation

- spontaneous infarction

- Sheehan's syndrome

- raised intracranial pressure

Box 1

\section{Clinical features of pituitary apoplexy}

- severe retro-orbital headache

- nausea and vomiting

- meningism, fever, impaired consciousness (subarachnoid blood)

- visual field defect, reduced visual acuity (upward expansion)

- ophthalmoplegia, commonly 3rd nerve palsy (lateral expansion)

- facial pain, numbness (lateral expansion)

\section{Box 2}

ophthalmological problems. Hypopituitarism may be transient or permanent, and formal endocrine testing is required to assess the need for replacement therapy. Long-term steroids are required in $66 \%$ of patients and thyroxine in $50 \% .^{5}$ Gonadotropin deficiency occurs in $58-76 \%$ of cases but does not always require replacement, and prolactin is elevated in $67-$ $100 \%$. Diabetes insipidus only affects $2-3 \%$.

\section{Comment}

Given the severity of the cardiac disease in our patient, he may well have had an unfavourable outcome even if his pituitary apoplexy had been diagnosed and the optimal treatment considered.

\section{Final diagnosis}

Pituitary apoplexy caused by haemorrhage into a previously asymptomatic adenoma, precipitated by thrombolytic therapy for myocardial infarction.

Keywords: pituitary apoplexy, acute myocardial infarction, adenoma

4 Glick RP, Tiesi JA. Subacute pituitary apoplexy: clinical and magnetic resonance imaging characteristics. Neurosurgery 1990; 27: $214-8$

5 Thorne MO. Anterior pituitary disorders. In: Weatherall DJ, Ledingham JG, Warrell DA, eds, Oxford textbook of medicine, 3rd edn. Oxford: Oxford Medical Publications, 1996. 